\title{
Distinct Alterations in Cerebellar Connectivity with Substantia Nigra and Ventral Tegmental Area in Parkinson's Disease
}

Ian M. O’Shea ${ }^{\mathrm{a}}$, Haroon S. Popal ${ }^{\mathrm{b}}$, Ingrid R. Olson ${ }^{\mathrm{c}}$, Vishnu P. Murty*d, David V. Smith*e *authors contributed equally to this work

\section{Affiliations}

[a-e]: Department of Psychology, Weiss Hall, Temple University, 1701 N. $13^{\text {th }}$ St, Philadelphia, PA 19112, USA

\section{Email addresses}
a. 1an.oshea@temple.edu
b. haroon.popal@temple.edu
c. ingrid.olson@temple.edu
d. vishnu.murty@temple.edu
e. david.v.smith@,temple.edu

\section{Correspondence}

David V. Smith (avid.v.smith@temple.edu) or Vishnu P. Murty (vishnu.murty@temple.edu) Department of Psychology, Temple University. 


\begin{abstract}
In Parkinson's disease (PD), neurodegeneration of dopaminergic neurons occurs in the midbrain, specifically targeting the substantia nigra (SN), while leaving the ventral tegmental area (VTA) relatively spared in early phases of the disease. Although the SN and VTA are known to be functionally dissociable in healthy adults, it remains unclear how this dissociation is altered in PD. To examine this issue, we performed a whole-brain analysis to compare functional coupling in PD to healthy adults using resting-state fMRI data compiled from three independent datasets. Our analysis showed that across the sample, the $\mathrm{SN}$ had greater coupling to the precuneus, anterior cingulate gyrus, and areas of the occipital cortex, partially replicating our previous work in healthy young adults. Notably, we also found that, in PD, VTA-right cerebellum coupling was higher than SN-right cerebellum coupling, whereas the opposite trend occurred in healthy controls. This double dissociation may reflect a compensatory role of the cerebellum in PD and could provide a potential target for future study and treatment.
\end{abstract}

Keywords: Parkinson's, midbrain, cerebellum, fMRI, dopamine 


\begin{abstract}
Abbreviations: PD: Parkinson's disease, SN: substantia nigra, VTA: ventral tegmental area, ROI: region of interest, rs-fMRI: resting state functional magnetic resonance imaging, MPRAGE: magnetization prepared rapid gradient echo sequence, TR: repetition time, TE: echo time, FOV: field of view, BOLD: blood-oxygen level-dependent, EPI: echo planar imaging
\end{abstract}

\title{
Introduction
}

The pathological process underlying Parkinson's Disease (PD) targets dopamine neurons in the midbrain. In early phases of the disease, neurodegeneration occurs in the dopaminergic neurons of the substantia nigra (SN), an area of the midbrain responsible for initiating movement through the nigrostriatal network. However, the dopaminergic neurons in the ventral tegmental area (VTA), a neighboring midbrain structure, are thought to be spared from the neuronal degradation in early stages of PD (Dagher \& Robbins, 2009; Damier et al., 1999; Fearnley \& Lees, 1991, Hirsch, Graybiel, \& Agid, 1988). These neurons play a pivotal role in the mesocortical and mesolimbic pathways, functional networks responsible for executive function, reward signaling, and motivation. The lack of degeneration in the VTA may explain why PD patients display motor deficits like bradykinesia and tremors but maintain the ability to engage in motivated behavior (Dagher and Robbins, 2009). This differentiation in behaviors that are affected and remain intact in early PD are thought to result from the discrete patterns of connectivity arising from the SN and VTA areas of the dopaminergic pathways. However, neuroimaging work has yet to investigate the way the SN and VTA differentially interact with the rest of the brain in PD compared to controls.

Within the midbrain, the SN and VTA perform separate yet parallel functions ranging from attention to learning to action (Berridge et al., 2009, Salamone et al., 2007, Wise, 2004). Previous work from our lab provides evidence of reliable differences in two distinct functional 
networks of the midbrain in controls (Murty, et al., 2014). The SN projected more strongly to sensorimotor areas of the cortex like the precentral gyrus while the VTA had greater connectivity to areas associated with reward and motivation such as the nucleus accumbens. The differentiation across these nuclei permits the ability for humans to perform motivated actions through widespread afferent and efferent circuits in the brain. Notably, other organizational schemas of the midbrain have challenged the notion of treating these two nuclei as distinct, but rather treat them as a unified structure varying across a continuous gradient (Bromberg-Martin et al., 2010; Düzel et al., 2009). However, PD is proposed to affect the SN to a greater extent than the VTA, highlighting a neurological condition that naturally draws a dissociation between these two networks.

A growing body of work has used resting-state functional magnetic resonance imaging (rs-fMRI) to study midbrain networks in PD, focusing on interactions of the SN with systems responsible for motor planning and execution (Hacker et al., 2012; Wu et al., 2012; Sharman et al., 2013. Previous work found that the SN has decreased functional connectivity to the supplementary motor area, default mode network, and dorsolateral prefrontal cortex in patients with PD, where in controls there is increased SN connectivity to these regions (Wu et al., 2012). Administration of Levadopa partially normalized these differences, indicating dopamine's role in regulating connectivity (Wu et al., 2012). Others suggest that $\mathrm{SN}$ connectivity is reduced to the thalamus, globus pallidus, and the putamen (Sharman et al., 2013). Futher investigation of the striatum indicated decreased striatal connectivity with the midbrain, however the SN and VTA were not studied individually (Hacker et al., 2012). This research with rs-fMRI focused on larger networks that contribute to motor functioning without examining the more granular interactions of sub-regions. Namely, this work did not include the VTA and its projections to mesocortical 
and mesolimbic systems, thus leaving open questions about how interactions between the SN and VTA may be altered by changes in dopaminergic tone.

The goal of the present study was to investigate dissociations between dopaminergic midbrain networks in patients with PD and healthy controls. The few studies that have examined this question used relatively small sample sizes (Hacker et al., 2012; Wu et al., 2012; Sharman et al., 2013), which may affect reproducibility of findings. Thus for the present study, we combined three open datasets of rs-fMRI data in order to obtain robust results. We first conducted a wholebrain analysis to analyze functional connectivity to the SN and VTA, which were defined by probabilistic atlases (Murty et al., 2014). Connectivity values were compared across these seed regions in whole-brain analyses to examine the differences between the two networks. We focused on the group by region of interest (ROI) interaction for network-specific effects of PD. To preview our findings, we find that PD differentially affects the two dopaminergic networks in the midbrain.

\section{Methods}

\section{Datasets}

Resting state functional magnetic resonance imaging (rs-fMRI) data were aggregated from three publicly available sources: 27 PD patients and 16 healthy controls from the NEUROCON project (Badea, et al., 2017), 20 PD patients and 20 controls from the Tao Wu group (Badea, et al., 2017), and 14 de novo PD patients and 14 controls from Tessa and colleagues. (Tessa, et al., 2019). In total, there were 111 participants: 61 PD patients and 50 healthy controls.

\section{Pre-registration}


The study was pre-registered using AsPredicted (https://aspredicted.org/blind.php?x=vs5zn6).

There were slight deviations in our methods from the pre-registration. Using whole-brain analysis deviated from the pre-registration, which indicated that an ROI-based analysis would be used. Given that this sample was made up of older adults - some of whom were diagnosed with PD — there may be different localizations of cortical ROIs due to cortical atrophy or thinning. Therefore the cortical ROIs from previous work in young adults were not used, and instead a whole-brain analysis was performed to ensure that no areas of significant connectivity were missed in the analysis.

\section{Image Acquisition}

Dataset 1 (Badea, et al., 2017): Neuroimaging data were collected using a 1.5 Tesla Siemens Avanto MRI scanner. Anatomical images were collected using a magnetization prepared rapid gradient echo sequence (MPRAGE) [Repetition time (TR): $1940 \mathrm{ms,} \mathrm{Echo} \mathrm{time} \mathrm{(TE):} 3.08$ ms, voxel size: $0.97 \times 0.97 \times 1 \mathrm{~mm}$, inversion time: data not available (N/A), field of view (FOV): N/A, matrix size: N/A]. Resting state images sensitive to blood-oxygen level-dependent (BOLD) contrast were collected using an echo planar imaging (EPI) sequence [TR: $3480 \mathrm{~ms}$, TE: $50 \mathrm{ms,}$ flip angle: 90 degrees, voxel size: 3.8 x 3.8 x 5 mm (without slice gaps), matrix size: 64 x 64 , number of slices: 27, FOV: N/A, volumes: 137 (8.05 min)].

Dataset 2 (Badea et al., 2017): Neuroimaging data were collected using a 3 Tesla Siemens Magnetom Trio scanner. Anatomical images were also collected using MPRAGE [TR: 1100 ms, TE: 3.39 ms, voxel size: 1 x 1 x $1 \mathrm{~mm}$, inversion time: N/A, FOV: N/A, matrix size: N/A]. Resting state images sensitive to BOLD contrast were collected using an EPI sequence [TR: $2 \mathrm{~s}$, TE: 40 ms, flip angle: 90 degrees, voxel size: 4 x 4 x 5 mm, matrix size: 64 x 64, number of slices: 28, FOV: 256 x $256 \mathrm{~mm}$, volumes: 239 (8 min)]. 
Dataset 3 (Tessa et al., 2019): Neuroimaging data were collected using a 1.5 Tesla Siemens Magneto Avanto MRI scanner. Anatomical images were collected using MPRAGE [TR: 1900 ms, TE: $3.44 \mathrm{~ms}$, inversion time: $1100 \mathrm{~ms}$, filp angle: 15 degrees, voxel size: 0.859 x 0.859 x $0.86 \mathrm{~mm}, \mathrm{FOV}: 220$ x 220mm, matrix size: 256 x 256]. Resting state fMRI images were collected using a T2-weighted EPI sequence [TR: $2130 \mathrm{~ms}$, TE: $40 \mathrm{~ms}$, flip angle: 90 degrees, voxel size: 4 x 4 x 5 mm, FOV: 256 x 256 mm, matrix size: 64 x 64, number of slices: 32, volumes: 230, interleaved slice acquisition].

\section{Neuroimaging Preprocessing}

All three datasets were downloaded from their respective sources in BIDS format and were preprocessed using fMRIPrep version 1.2.6-1, an image processing pipeline based on Nipype 1.4.2 (Esteban et al., 2019; Gorgolewski et al., 2011; Gorgolewski et al., 2017). The details below were adapted from the fMRIPrep preprocessing details with extraneous details being omitted for clarity. Importantly, data from all datasets were preprocessed using the same pipeline.

The T1w image was corrected for intensity non-uniformity (INU) with N4BiasFieldCorrection (Tustison et al. 2010), distributed with ANTs 2.2.0 (Avants et al. 2008), and used as T1w-reference throughout the workflow. Skull-stripping was then performed on the T1w-reference using a Nipype implementation of the antsBrainExtraction.sh workflow (from ANTs), with OASIS30ANTs as the target template. Brain tissue segmentation of cerebrospinal fluid (CSF), white-matter (WM) and gray-matter (GM) was performed on the brain-extracted T1w using fast (FSL 5.0.9, Zhang, Brady, and Smith 2001). Volume-based spatial normalization to MNI152NLin2009cAsym standard space was performed through nonlinear registration with antsRegistration (ANTs 2.2.0), using brain-extracted versions of both $\mathrm{T} 1 \mathrm{w}$ reference and the 
T1w template. To this end, the ICBM 152 Nonlinear Asymmetrical template version 2009c

(Fonov et al. 2009) template was selected for spatial normalization.

For each of the BOLD runs contained per subject, the following preprocessing steps were performed. First, a reference volume and its skull-stripped version were generated using a custom methodology of fMRIPrep. Head-motion parameters with respect to the BOLD reference (transformation matrices, and six corresponding rotation and translation parameters) were estimated before any spatiotemporal filtering using mcflirt (FSL 5.0.9, Jenkinson et al. 2002). BOLD runs were slice-time corrected using 3dTshift from AFNI 20160207 (Cox 1996; Cox and Hyde 1997). Based on the estimated susceptibility distortion, a corrected EPI reference was calculated for a more accurate co-registration with the anatomical reference. The BOLD reference was then co-registered to the T1w reference using flirt (FSL 5.0.9, Jenkinson and Smith 2001) with the boundary-based registration (Greve and Fischl 2009) cost-function. Coregistration was configured with nine degrees of freedom to account for distortions remaining in the BOLD reference. The BOLD time-series (including slice-timing correction when applied) were resampled onto their original, native space by applying a single, composite transform to correct for head-motion and susceptibility distortions. These resampled BOLD time-series will be referred to as preprocessed BOLD in original space, or just preprocessed BOLD. The BOLD time-series were resampled into standard space, generating a preprocessed BOLD run in MNI152NLin2009cAsym space.

Automatic identification of motion artifacts using independent component analysis (ICAAROMA, Pruim et al. 2015) was performed on the preprocessed BOLD on MNI space timeseries after removal of non-steady state volumes and spatial smoothing with an isotropic, Gaussian kernel of 6mm FWHM (full-width half-maximum). AROMA motion components were 
subsequently included as regressors in our analyses (see below). Additional confounding timeseries were calculated based on the preprocessed BOLD: framewise displacement (FD) and three regional signals (cerebral spinal fluid, white matter, and grey matter). FD was computed using the relative root mean square displacement between affines (Jenkinson et al. 2002). The three global signals are extracted within the CSF, the WM, and the whole-brain masks. Although, we note that FD was not used for "scrubbing" (Power et al., 2012; Power et al., 2014). All resamplings were performed with a single interpolation step by composing all the pertinent transformations (i.e., head-motion transform matrices, susceptibility distortion correction when available, and co-registrations to anatomical and output spaces). Gridded (volumetric) resamplings were performed using antsApplyTransforms (ANTs), configured with Lanczos interpolation to minimize the smoothing effects of other kernels (Lanczos 1964).

We removed subjects based on the Entropy Focus criterion (efc), Foreground to Background Energy Ratio (fber), Temporal Signal to Noise Ratio (tsnr), average framewise displacement (fd_mean), and Ghost to Signal Ratio in the Y direction (gsr_y) Image Quality Metrics (IQMs) from MRIQC (Esteban, et al., 2017). Outlier runs were defined as runs with efc, fd_mean, or gsr_y values exceeding 1.5 times the inter-quartile range above the 75 th percentile, as well as those with fber and tsnr values lower than 1.5 times the lower bound minus the 25 th percentile (i.e., a boxplot threshold). Using these parameters, 8 subjects were excluded for a total sample of 103 participants: 55 PD patients and 48 healthy controls.

\section{First-level Analysis}

Whole-brain analysis was performed across the three datasets using the FMRI Expert Analysis Tool (FEAT) from the FMRIB Software Library (FSL) to determine differences in functional coupling of midbrain regions between healthy controls and PD patients. Seed regions, 
including the substantia nigra (SN) and ventral tegmental area (VTA), were defined using probabilistic atlases from previous work (Murty, et al., 2014). These regions were then resampled to fit the dimensions of each of the 3 datasets using AFNI's 3dResample (Cox, 1996).

First, the eigenvariate of BOLD signaling for a single time series was extracted for each seed ROI within each subject, using a weighted average based on each probabilistic atlas. Then, FEAT was used to create a general linear model (GLM) with local correction for autocorrelation and regressors for the SN and VTA. This GLM created brain maps of FC within each subject for voxels predicted by the regressors of the following of contrasts of interest: $\mathrm{SN}>$ baseline, VTA $>$ baseline, SN $>$ VTA, and VTA $>$ SN. The GLM also accounted for confound regressors of WM and CSF signal, non-steady-state volumes, cosine basis functions (to highpass filter with 128s cutoff), and ICA-AROMA motion.

\section{Higher-level Analyses}

For subjects of the NEUROCON dataset with two imaging runs, a second analysis was performed using a fixed effects model to create average BOLD maps across the runs for each subject. All brain maps were then used in a higher level random effects GLM analysis to compare functional coupling of the entire sample across multiple regressors: group (con > PD, PD $>$ con), ROI (SN > VTA, VTA > SN), and group by ROI interactions (Woolrich et al., 2004). This analysis utilized the Randomise function of FSL (Winkler et al., 2014) with Threshold-Free Cluster Enhancement (TFCE). To facilitate integration across datasets, our group-level model also included covariates for age, sex, dataset, efc, fber, tsnr, fd_mean, and gsr_y.

Any clusters of significant functional coupling from the group by ROI interaction were further investigated by calculating an average value of functional coupling across time points (t- 
stat) from both ROIs to that cluster in each subject. Then, two-tailed paired sample t-tests were run using $\mathrm{R}$ version 4.0.3 and RStudio (RStudio Team, 2020, version 1.3.1093) to determine within-group and within-ROI effects. For all statistical analyses, a significance threshold of $\mathrm{p}<$ 0.05 was used. For clusters in the cerebellum, a flat-map was created using the spatially unbiased atlas template of the cerebellum and brainstem (SUIT) toolbox (Diedrichsen \& Zotow, 2015). The anatomical location of the clusters could then compared to the functional boundaries defined in recent work to infer its contributions to functioning (King et al., 2019). Any clusters were also input to LittleBrain to further interpret functional implications (Guell et al., 2019).

\section{Results}

First, we wanted to determine the differences in functional coupling of the SN compared to the VTA, collapsing across group. Whole-brain analysis showed a significant effect of ROI ( $p$ $<0.05$, whole-brain corrected), such that the SN had greater coupling than the VTA to various regions throughout the cortex, including the precuneus, anterior cingulate gyrus, and areas of the occipital cortex, partially replicating previous results (Figure 1; Table 1; Murty et al., 2014). However, unlike prior reports in younger adult populations, the reverse contrast of VTA greater than SN did not show any significant differences at our correction threshold. 


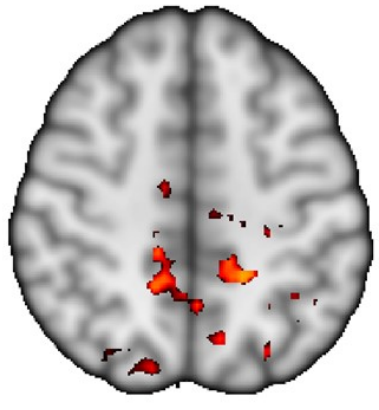

$\mathrm{Z}=50$

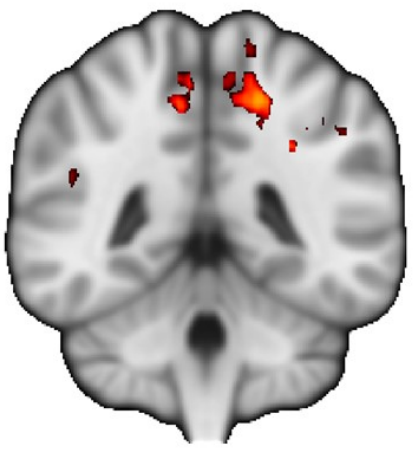

$\mathrm{Y}=-44$

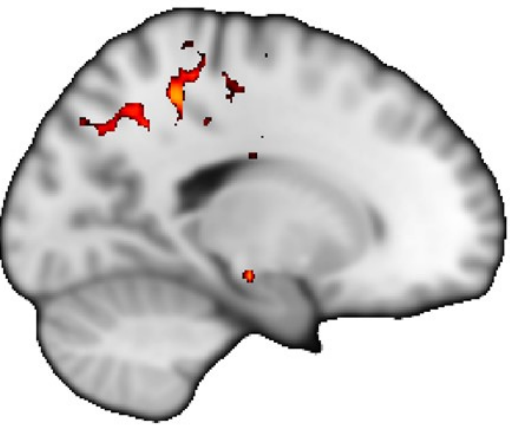

$X=-18$

Figure 1. Whole-brain connectivity analysis reveals a main effect of $\mathrm{SN}>\mathrm{VTA}$. Image is thresholded at $\mathrm{p}<0.05$

Coordinates correspond with peak activation of the largest cluster, in the precuneus cortex.

\begin{tabular}{|c|c|c|c|c|c|}
\hline Region & Voxels & Peak activation & $\mathbf{x}$ & $\mathbf{y}$ & $\mathbf{z}$ \\
\hline Precuneus Cortex (left) & 2056 & 0.981 & -18 & -44 & 50 \\
\hline Brain-stem (right SN) & 291 & 1 & 12 & -24 & -20 \\
\hline Brain-stem (left SN) & 241 & 1 & -10 & -20 & -16 \\
\hline $\begin{array}{l}\text { Cingulate Gyrus, anterior } \\
\text { division (left) }\end{array}$ & 235 & 0.981 & -4 & -8 & 30 \\
\hline $\begin{array}{l}\text { Lateral Occipital Cortex, } \\
\text { superior division (right) }\end{array}$ & 173 & 0.973 & 18 & -76 & 54 \\
\hline $\begin{array}{l}\text { Supramarginal Gryus, } \\
\text { posterior division/Angular } \\
\text { Gyrus (right) }\end{array}$ & 100 & 0.967 & 48 & -42 & 24 \\
\hline $\begin{array}{l}\text { Supramarginal Gyrus, anterior } \\
\text { division (right) }\end{array}$ & 65 & 0.964 & 68 & -26 & 34 \\
\hline Angular Gyrus (left) & 53 & 0.962 & -44 & -56 & 16 \\
\hline $\begin{array}{l}\text { Lateral Occipital Cortex, } \\
\text { superior division (left) }\end{array}$ & 34 & 0.955 & -32 & -60 & 54 \\
\hline
\end{tabular}




\begin{tabular}{|c|c|c|c|c|c|}
\hline Cerebral White Matter (left) & 20 & 0.964 & -16 & -14 & 28 \\
\hline $\begin{array}{l}\text { Lateral Occipital Cortex, } \\
\text { superior division (right) }\end{array}$ & 12 & 0.954 & 46 & -72 & 18 \\
\hline Superior Frontal Gyrus (left) & 12 & 0.955 & -14 & -10 & 68 \\
\hline $\begin{array}{l}\text { Cingulate Gyrus, anterior } \\
\text { division (right) }\end{array}$ & 10 & 0.956 & 6 & 4 & 30 \\
\hline $\begin{array}{l}\text { Lateral Occipital Cortex, } \\
\text { superior division (left) }\end{array}$ & 10 & 0.953 & -26 & -64 & 46 \\
\hline
\end{tabular}

Table 1. Voxel clusters where connectivity is greater to SN than VTA. The table lists region name, amount of voxels, peak activation, and coordinates in MNI space for clusters significant at a $p<0.05$ threshold. Clusters of less than 10 voxels ( 8 clusters) were removed from the table.

Next, we wanted to determine differences in coupling between PD patients and the healthy controls, collapsing across seed ROIs. Again, a whole-brain analysis was performed to identify regions whose coupling differed across the two groups. There were no main effects of group, such that collapsed across ROI, functional coupling did not differ when comparing the PD group to the controls.

Finally, we wanted to determine if there were any significant interactions between group and ROI to investigate whether PD had a region-specific effect on functional coupling. Wholebrain analysis was performed, which indicated a significant group by ROI interaction in the right cerebellum $(\mathrm{p}<0.05$, whole-brain corrected; Figure 2A-B). Post-hoc analyses revealed that in PD patients, the VTA had enhanced functional coupling with a region in the right cerebellar cortex (e.g. VIIB and VIIIA) compared to the $\mathrm{SN}(\mathrm{t}=-5.20, \mathrm{p}<0.001,95 \% \mathrm{CI}=[-0.80,-0.36])$, while in healthy controls there was greater SN than VTA coupling with the right cerebellar cortex $(\mathrm{t}=$ $4.69, \mathrm{p}<0.001,95 \% \mathrm{CI}=[0.31,0.77]$; Figure $2 \mathrm{C})$. In the $\mathrm{SN}$, contralateral and ipsilateral 
connectivity to the right cerebellar cortex were not significantly different. Effects of laterality were not investigated in the VTA due to its medial location in the brain.
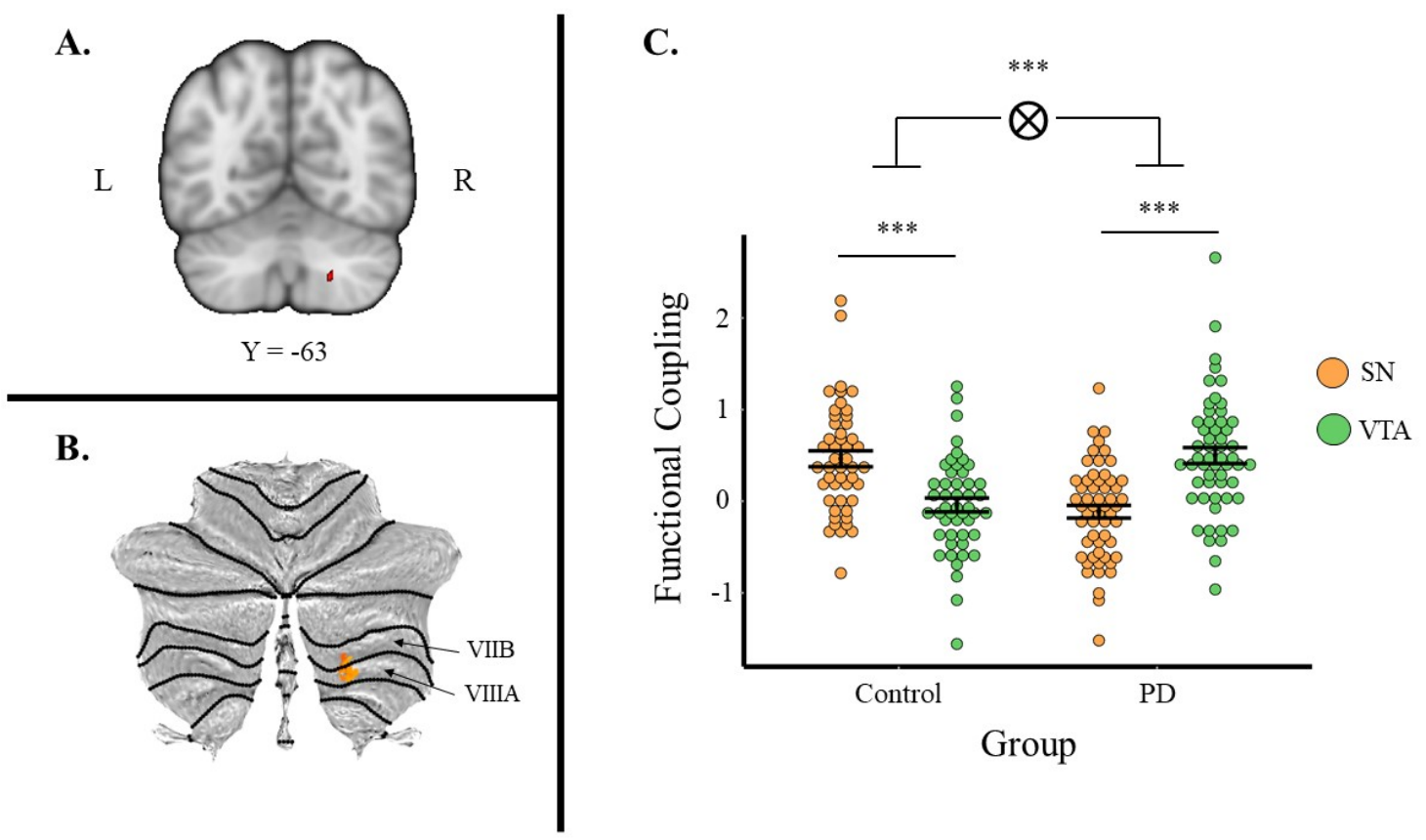

Figure 2. Whole-brain connectivity analysis reveals group by ROI interaction in right cerebellum. A. Axial view of brain showing cerebellar cluster, thresholded at $\mathrm{p}<0.05$. B. The same cluster as in A., but flat-mapped, shows that the region corresponds to VIIB and VIIIA regions of the cerebellum; and C. Dotplot reveals direction of interaction, where the PD group had greater VTA than SN coupling with the right cerebellum and the control group had greater SN than VTA coupling to the right cerebellum. $(\mathrm{p}<0.001 * * *)$

\section{Discussion}

Using three publicly available rs-fMRI datasets, we provide novel evidence of differential connectivity of the SN and VTA in Parkinson's disease. Namely, we find that SN functional coupling with motor and pre-motor cortical networks remains intact across PD and control groups. However, we were surprised to find a prominent double disassociation of the midbrain connectivity with the right cerebellum, such that in Parkinson's, there was greater VTA-right cerebellum coupling as compared to the SN-right cerebellum — while in healthy controls, there 
was greater SN-right cerebellum coupling as compared to VTA-right cerebellum. Together, these findings suggest an important differentiation of connectivity of the dopaminergic midbrain with the cerebellum, when taking into account functional heterogeneity across the midbrain.

Although the cerebellar findings were unexpected, they are not without precedent. Projections from the deep cerebellar nuclei to the VTA are monosynaptic and bidirectional. D'Ambra and colleagues (2021) showed that microstimulation of the deep cerebellar nuclei in mice modulated nucleus accumbens spiking activity, both excitatory and inhibitory, depending on the particular location recorded from within the nucleus accumbens (D'Ambra et al., 2021). Another study found that optogenetic stimulation of cerebellar-VTA axons dramatically altered goal-oriented behavior (Carta et al., 2019). In humans, this circuit is considered to be part of a larger, highly integrated learning system (Caligiore et al., 2019). In PD, the role of the cerebellum is relatively unclear, however Wu \& Hallett (2013) suggest that the structure has both pathological and compensatory effects. These compensatory effects are thought to help maintain both motor and cognitive function to make up for the degeneration of dopaminergic neurons in the SN (Wu \& Hallett, 2013). Therefore, increased VTA-cerebellum coupling may be the result of the cerebellum overcompensating for neurodegeneration in the $\mathrm{SN}$, while the relatively decreased SN-cerebellum coupling in PD could be indicative of a pathological effect. In line with this interpretation, reward magnitude has been shown to correlate with cerebellar activity in PD, while correlating with activity of prefrontal and rhinal cortices and the thalamus in controls (Goerendt et al., 2004). Thus, these findings correspond with motivated learning being mostly spared in PD while motor function deteriorates (Dagher and Robbins, 2009).

The specificity of midbrain-cerebellar connectivity may give insight into the functional implications of our findings. Dissociations in midbrain connectivity with the cerebellum spanned 
cerebellar lobules VIIB and VIIIA. Based on the functional boundaries defined by King et al. (2019), a study of cerebellar contributions to cognition, these clusters correspond to executivefunction related regions of the cerebellum that are known to contribute to divided attention (King et al., 2019). Similarly, using LittleBrain, we found that the clusters correspond to cerebellar voxels that project to dorsal and ventral attention networks at rest, critically reflecting that the cerebellar region is involved in cognitive, not motor processes (Guell et al., 2019). Given that executive functioning is spared in the early phases of PD, cerebellar engagement with the VTA may represent how the cerebellum maintains executive functioning through a goal-oriented compensatory mechanism. However, future studies relating these connectivity profiles with executive function deficits are necessary to confirm these hypotheses. Furthermore, late-stage executive deficits in PD may reflect the inability of the cerebellum to keep up with dopaminergic degradation. Ultimately, these results indicate the cerebellum as a possible target of treatment for PD in the future and support further investigation into how cerebellar subregions are impacted by dopaminergic degeneration.

Our findings failed to replicate many of the findings previously shown regarding SN connectivity using rs-fMRI in PD. Namely, we did not find deficits in SN connectivity to supplementary motor area, dorsolateral prefrontal cortex, thalamus, default mode network nodes, or striatal structures in PD, as have previous groups (Hacker et al., 2012; Wu et al., 2012; Sharman et al., 2013). In fact, we found a similar pattern of connectivity of the SN when compared to the VTA with motor and pre-motor cortical regions that did not differ across groups. One factor that could explain this lack of replication is medication status. Wu and colleagues (2012) showed that deficits between the SN and cortical targets in motor and executive regions were ameliorated with Levadopa administration (Wu et al., 2012). In our 
study, we collapsed across three datasets in which PD patients were un-medicated or medicated with L-Dopa. Medication status was not evenly distributed across our samples, and thus we could not effectively control for this factor in our analysis. Despite controlling for dataset as a confound in our GLM, these medication differences may have influenced results; specifically, Levadopa administration may have compensated for any SN connectivity deficits. Future research should study how Levadopa affects the dissociation between the VTA and SN networks in PD.

Outside of group-related differences, when collapsing across the entire sample, SN connectivity was higher than VTA connectivity to the precuneus, anterior cingulate gyrus, and portions of the occipital cortex. This finding, in part, replicates SN connectivity from a previous study in our lab conducted in a younger adult population (Murty et al., 2014). Unlike our prior study, however, the VTA did not show enhanced connectivity with the nucleus accumbens and subgenual cingulate when compared to the SN, conflicting with previous findings. One reason we may have failed to replicate these findings may be the differences in age across samples. Our original study was conducted in a sample of younger adults aged 18 to 25 , whereas our current sample represents older adults aged 36 to 86 . Critically, prior research across rodents and humans has shown decreases in dopaminergic neuromodulation throughout aging, which could alter the connectivity of the VTA at the population level (Bäckman et al., 2006, Dreher et al., 2008).

Overall, we provide corroborating evidence that there are two dissociable midbrain networks, stemming from the SN and VTA, respectively. Not only does the dissociation provide a better understanding of PD, it also implies that these two regions contribute to cognition and behavior differently via their distinct connections throughout the brain. This observation has 
implications for other disorders like schizophrenia, substance abuse, ADHD, and depression, disorders in which the pathophysiology is related to dopamine dysfunction. We also provide evidence that the cerebellum may play a significant role in regulating these pathways, consistent with recent work in mice (D'Ambra et al., 2021) and therefore in the development of neuropsychiatric conditions. A growing body of work links mood disorders such as depression to dysregulated functional connectivity within the cerebellum (Frazier et al., 2021; Tepfer et al., 2021). Of note, depression is common in PD (Reijnders et al., 2008); whether this can be linked to common pathways is a question for future research. Overall, our study provides a foundation for future translational work by furthering our understanding of the midbrain's role in cognition and motor control and characterizing how dysregulated cerebellar connectivity contributes to PD. 


\section{Funding}

This work was supported, in part, by National Institutes of Health grants RF1-AG067011 (DVS), R03-DA046733 (DVS), R21-DA043568 (VPM), and K01- MH111991 (VPM).

\section{Conflict of interest statement}

The authors have no conflicts to disclose.

\section{Acknowledgments}

We would like to thank the NEUROCON project, the Tao Wu group, and Tessa and colleagues for making their data publically available. We note that this work has been deposited on PsyArXiv as a preprint. We note that DVS was a Research Fellow of the Public Policy Lab at Temple University during the preparation of this manuscript (2019-2020 academic year).

\section{References}

Avants, B. B., Epstein, C. L., Grossman, M., \& Gee, J. C. (2008). Symmetric diffeomorphic image registration with cross-correlation: Evaluating automated labeling of elderly and neurodegenerative brain. Medical Image Analysis, 12(1), 26-41. https://doi.org/10.1016/j.media.2007.06.004

Bäckman, L., Nyberg, L., Lindenberger, U., Li, S. C., \& Farde, L. (2006). The correlative triad among aging, dopamine, and cognition: Current status and future prospects. In Neuroscience and Biobehavioral Reviews (Vol. 30, Issue 6, pp. 791-807). Pergamon.

https://doi.org/10.1016/j.neubiorev.2006.06.005

Badea, L., Onu, M., Wu, T., Roceanu, A., \& Bajenaru, O. (2017). Exploring the reproducibility of functional connectivity alterations in Parkinson's disease. https://doi.org/10.1371/journal.pone.0188196

Berridge, K. C., Robinson, T. E., \& Aldridge, J. W. (2009). Dissecting components of reward: "liking", "wanting", and learning. In Current Opinion in Pharmacology (Vol. 9, Issue 1, pp. 6573). Elsevier. https://doi.org/10.1016/j.coph.2008.12.014

Bromberg-Martin, E. S., Matsumoto, M., \& Hikosaka, O. (2010). Dopamine in Motivational Control: Rewarding, Aversive, and Alerting. In Neuron (Vol. 68, Issue 5, pp. 815-834). Cell Press. https://doi.org/10.1016/j.neuron.2010.11.022 
Caligiore, D., Arbib, M. A., Miall, R. C., \& Baldassarre, G. (2019). The super-learning hypothesis: Integrating learning processes across cortex, cerebellum and basal ganglia. In Neuroscience and Biobehavioral Reviews (Vol. 100, pp. 19-34). Elsevier Ltd. https://doi.org/10.1016/j.neubiorev.2019.02.008

Carta, I., Chen, C. H., Schott, A. L., Dorizan, S., \& Khodakhah, K. (2019). Cerebellar modulation of the reward circuitry and social behavior. Science, 363(6424). https://doi.org/10.1126/science.aav0581

Cox, R. W. (1996). AFNI: Software for analysis and visualization of functional magnetic resonance neuroimages. Computers and Biomedical Research, 29(3), 162-173. https://doi.org/10.1006/cbmr.1996.0014

Cox, R. W., \& Hyde, J. S. (1997). Software Tools for Analysis and Visualization of FMRI Data. NMR in Biomedicine, 10: 171-178

Dagher, A., \& Robbins, T. W. (2009). Personality, Addiction, Dopamine: Insights from Parkinson's Disease. In Neuron (Vol. 61, Issue 4, pp. 502-510). Cell Press. https://doi.org/10.1016/j.neuron.2009.01.031

D’Ambra, A. F., Jung, S. J., Ganesan S., Antzoulatos E. G., Fioravante D. (2021- submitted). Cerebellar Activation Bidirectionally Regulates Nucleus Accumbens Medial Shell and Core. bioRxiv.

Damier, P., Hirsch, E. C., Agid, Y., \& Graybiel, A. M. (1999). The substantia nigra of the human brain II. Patterns of loss of dopamine-containing neurons in Parkinson's disease. In Brain (Vol. 122).

Diedrichsen, J., \& Zotow, E. (2015). Surface-Based Display of Volume-Averaged Cerebellar Imaging Data. https://doi.org/10.1371/journal.pone.0133402

Dreher, J. C., Meyer-Lindenberg, A., Kohn, P., \& Berman, K. F. (2008). Age-related changes in midbrain dopaminergic regulation of the human reward system. Proceedings of the National Academy of Sciences, 105(39), 15106-15111.

Düzel, E., Bunzeck, N., Guitart-Masip, M., Wittmann, B., Schott, B. H., \& Tobler, P. N. (2009). Functional imaging of the human dopaminergic midbrain. Trends in Neurosciences, 32(6), 321328. https://doi.org/10.1016/j.tins.2009.02.005

Esteban, O., Birman, D., Schaer, M., Koyejo, O. O., Poldrack, R. A., \& Gorgolewski, K. J. (2017). MRIQC: Advancing the automatic prediction of image quality in MRI from unseen sites. https://doi.org/10.1371/journal.pone.0184661

Esteban, O., Markiewicz, C. J., Blair, R. W., Moodie, C. A., Isik, A. I., Erramuzpe, A., Kent, J. D., Goncalves, M., DuPre, E., Snyder, M., Oya, H., Ghosh, S. S., Wright, J., Durnez, J., Poldrack, R. A., \& Gorgolewski, K. J. (2019). fMRIPrep: a robust preprocessing pipeline for functional MRI. Nature Methods, 16(1), 111-116. https://doi.org/10.1038/s41592-018-0235-4 
Fearnley, J. M., \& Lees, A. J. (1991). Ageing and parkinson's disease: Substantia nigra regional selectivity. Brain, 114(5), 2283-2301. https://doi.org/10.1093/brain/114.5.2283

Fonov, V., Evans, A., McKinstry, R., Almli, C., \& Collins, D. (2009). Unbiased nonlinear average age-appropriate brain templates from birth to adulthood. NeuroImage, 47, S102. https://doi.org/10.1016/s1053-8119(09)70884-5

Frazier, M., Hoffman L. J., Sullivan-Toole, H., Olino, T. M., Olson, I. R. (2021-submitted). A missing link in affect regulation: The cerebellum.

Goerendt, I. K., Lawrence, A. D., \& Brooks, D. J. (2004). Reward Processing in Health and Parkinson's Disease: Neural Organization and Reorganization. Cortex, 14, 73-80.

https://doi.org/10.1093/cercor/bhg105

Gorgolewski, K., Burns, C. D., Madison, C., Clark, D., Halchenko, Y. O., Waskom, M. L., \& Ghosh, S. S. (2011). Nipype: A flexible, lightweight and extensible neuroimaging data processing framework in Python. Frontiers in Neuroinformatics, 5. https://doi.org/10.3389/fninf.2011.00013

Gorgolewski, K. J., Esteban, O., Ellis, D. G., Notter, M. P., Ziegler, E., Johnson, H., Hamalainen, C., Yvernault, B., Burns, C., Manhães-Savio, A., Jarecka, D., Markiewicz, C. J., Salo, T., Clark, D., Waskom, M., Wong, J., Modat, M., Dewey, B. E., Clark, M. G., ... Ghosh, S. (2017). Nipype: a flexible, lightweight and extensible neuroimaging data processing framework in Python. 0.13.1. https://doi.org/10.5281/ZENODO.581704

Greve, D. N., \& Fischl, B. (2009). Accurate and robust brain image alignment using boundarybased registration. NeuroImage, 48(1), 63-72. https://doi.org/10.1016/j.neuroimage.2009.06.060

Guell, X., Goncalves, M., Kaczmarzyk, J. R., Gabrieli, J. D. E., Schmahmann, J. D., \& Ghoshid, S. S. (2019). LittleBrain: A gradient-based tool for the topographical interpretation of cerebellar neuroimaging findings. https://doi.org/10.1371/journal.pone.0210028

Hacker, C. D., Perlmutter, J. S., Criswell, S. R., Ances, B. M., \& Snyder, A. Z. (2012). Resting state functional connectivity of the striatum in Parkinson's disease. Brain, 135(12), 3699-3711. https://doi.org/10.1093/brain/aws281

Hirsch, E., Graybielt, A. M., \& Agid, Y. A. (1986). 28. National Research Council Nutrient Requirements of Beef Cattle Sixth revised edn Nutrient Requirements of Domestic Animals Number 4. In 16. Serengeti Ecological Monitoring Programme, Serengeti Wildlife Research Centre, PO Box (Vol. 17, Issue 2). Iowa State Univ. Press. Ames.

Id, C. T., Toschi, N., Id, S. O., Valenza, G., Lucetti, C., Barbieri, R., \& Diciotti, S. (2019). Central modulation of parasympathetic outflow is impaired in de novo Parkinson's disease patients. https://doi.org/10.1371/journal.pone.0210324

Jenkinson, M. (2003). Fast, automated, N-dimensional phase-unwrapping algorithm. Magnetic Resonance in Medicine, 49(1), 193-197. https://doi.org/10.1002/mrm.10354 
Jenkinson, M., Bannister, P., Brady, M., \& Smith, S. (2002). Improved Optimization for the Robust and Accurate Linear Registration and Motion Correction of Brain Images. NeuroImage, 17(2), 825-841. https://doi.org/10.1006/nimg.2002.1132

Jenkinson, M., \& Smith, S. (2001). A global optimisation method for robust affine registration of brain images. Medical Image Analysis, 5(2), 143-156. https://doi.org/10.1016/S13618415(01)00036-6

King, M., Hernandez-Castillo, C. R., Poldrack, R. A., Ivry, R. B., \& Diedrichsen, J. (2019). Functional boundaries in the human cerebellum revealed by a multi-domain task battery. Nature Neuroscience, 22(8), 1371-1378. https://doi.org/10.1038/s41593-019-0436-X

Lanczos, C. (1964). EVALUATION OF NOISY DATA*. In ANAL. Ser. B. https://epubs.siam.org/page/terms

Murty, V. P., Shermohammed, M., Smith, D. V., Carter, R. M. K., Huettel, S. A., \& Adcock, R. A. (2014). Resting state networks distinguish human ventral tegmental area from substantia nigra. NeuroImage, 100, 580-589. https://doi.org/10.1016/j.neuroimage.2014.06.047

Power, J. D., Barnes, K. A., Snyder, A. Z., Schlaggar, B. L., \& Petersen, S. E. (2012). Spurious but systematic correlations in functional connectivity MRI networks arise from subject motion. NeuroImage, 59(3), 2142-2154. https://doi.org/10.1016/j.neuroimage.2011.10.018

Power, J. D., Mitra, A., Laumann, T. O., Snyder, A. Z., Schlaggar, B. L., \& Petersen, S. E. (2014). Methods to detect, characterize, and remove motion artifact in resting state fMRI. NeuroImage, 84, 320-341. https://doi.org/10.1016/j.neuroimage.2013.08.048

Pruim, R. H. R., Mennes, M., van Rooij, D., Llera, A., Buitelaar, J. K., \& Beckmann, C. F. (2015). ICA-AROMA: A robust ICA-based strategy for removing motion artifacts from fMRI data. NeuroImage, 112, 267-277. https://doi.org/10.1016/j.neuroimage.2015.02.064

Reijnders, J. S. A. M., Ehrt, U., Weber, W. E. J., Aarsland, D., \& Leentjens, A. F. G. (2008). A systematic review of prevalence studies of depression in Parkinson's disease. In Movement Disorders (Vol. 23, Issue 2, pp. 183-189). https://doi.org/10.1002/mds.21803

Salamone, J. D., Correa, M., Farrar, A., \& Mingote, S. M. (2007). Effort-related functions of nucleus accumbens dopamine and associated forebrain circuits. In Psychopharmacology (Vol. 191, Issue 3, pp. 461-482). Springer. https://doi.org/10.1007/s00213-006-0668-9

Sharman, M., Valabregue, R., Perlbarg, V., Marrakchi-Kacem, L., Vidailhet, M., Benali, H., Brice, A., \& Lehéricy, S. (2013). Parkinson's disease patients show reduced cortical-subcortical sensorimotor connectivity. Movement Disorders, 28(4), 447-454.

https://doi.org/10.1002/mds.25255

Tepfer, L. J., Alloy, L. B., \& Smith, D. V. (2021). Family history of depression is associated with alterations in task-dependent connectivity between the cerebellum and ventromedial prefrontal cortex. Depression and Anxiety, 38(5). https://doi.org/10.1002/da.23143 
Tustison, N. J., Avants, B. B., Cook, P. A., Zheng, Y., Egan, A., Yushkevich, P. A., \& Gee, J. C. (2010). N4ITK: Improved N3 bias correction. IEEE Transactions on Medical Imaging, 29(6), 1310-1320. https://doi.org/10.1109/TMI.2010.2046908

Winkler A. M., Ridgway G. R., Webster M. A., Smith S. M., Nichols T. E. (2014). Permutation inference for the general linear model. NeuroImage, 2014;92:381-397

Wise, R. A. (2004). Dopamine, learning and motivation. In Nature Reviews Neuroscience (Vol. 5, Issue 6, pp. 483-494). Nature Publishing Group. https://doi.org/10.1038/nrn1406

Woolrich, M. W., Behrens, T. E. J., Beckmann, C. F., Jenkinson, M., \& Smith, S. M. (2004). Multilevel linear modelling for FMRI group analysis using Bayesian inference. NeuroImage, 21(4), 1732-1747. https://doi.org/10.1016/j.neuroimage.2003.12.023

Wu, T., \& Hallett, M. (2013). The cerebellum in Parkinson's disease. In Brain (Vol. 136, Issue 3, pp. 696-709). Oxford University Press. https://doi.org/10.1093/brain/aws360

Zhang, Y., Brady, M., \& Smith, S. (2001). Segmentation of brain MR images through a hidden Markov random field model and the expectation-maximization algorithm. IEEE Transactions on Medical Imaging, 20(1), 45-57. https://doi.org/10.1109/42.906424 